\title{
Correlating the patterns of Diabetic macular edema, Optical Coherence Tomography Biomarkers and Grade of Diabetic Retinopathy With Stage of Renal Disease
}

\section{Manisha Agarwal ( $\nabla$ agarwalmannii@yahoo.co.in )}

Dr Shroff's Charity Eye Hospital Delhi https://orcid.org/0000-0002-2277-001X

\section{Mani Sachdeva}

Dr Shroff's Charity Eye Hospital Delhi

\section{Shalin Shah}

Dr Shroff's Charity Eye Hospital Delhi

\section{Rajiv Raman}

Sankara Nethralaya

Padmaja Kumari Rani

LV Prasad Eye Institute

\section{Ramachandran Rajalakshmi}

Dr Mohan's Diabetes Specialities Centre Gopalapuram

\section{Sobha Sivaprasad}

Moorfields Eye Hospital NHS Foundation Trust

\section{T.P. Vignesh}

Aravind Eye Hospital

\section{Kim Ramasamy}

Aravind Eye Hospital

\section{Aishwarya Madharia}

Shri Sadguru Seva Sangh Trust

\section{Alok Sen}

Shri Sadguru Seva Sangh Trust

\section{Shalini Sugumar}

LV Prasad Eye Institute Bhubaneswar Campus Umesh Chandra Behera

LV Prasad Eye Institute Bhubaneswar Campus

Ann Maria Rodrigues

Giridhar Eye Institute

Giridhar Anantharaman

Giridhar Eye Institute 


\section{Swathi Priya}

Sankara Nethralaya

Atanu Majumdar

Dr Shroff's Charity Eye Hospital Delhi

\section{Research Article}

Keywords: Morphological pattern, DME, CKD, eGFR, OCT Biomarkers

Posted Date: December 3rd, 2021

DOl: https://doi.org/10.21203/rs.3.rs-868923/v1

License: (c) (1) This work is licensed under a Creative Commons Attribution 4.0 International License. Read Full License 


\section{Abstract}

\section{Purpose}

To correlate Optical coherence tomography (OCT) based morphological patterns of diabetic macular edema (DME), prognostic biomarkers and grade of diabetic retinopathy (DR) in patients with various stages of chronic kidney disease (CKD) secondary to diabetes.

\section{Design}

Multicentric retrospective cross-sectional study conducted at seven centres across India.

Methods

Data from medical records of patients with DME and CKD was entered in a common excel sheet across all seven centers. Staging of CKD was based on estimated glomerular filtration rate (eGFR).

\section{$\underline{\text { Results }}$}

The most common morphological pattern of DME was cystoid pattern (42\%) followed by the mixed pattern (31\%). The proportion of different morphological patterns did not significantly vary across various CKD stages $(p=0.836)$. Presence of external limiting membrane-ellipsoid zone (ELM-EZ) defects $(p<0.001)$ and foveal sub-field thickness $(p=0.024)$ showed a direct correlation with the stage of CKD which was statistically significant. Presence of hyper reflective dots (HRD) and disorganization of inner retinal layers (DRIL) showed no significant correlation with the stage of CKD. Sight threatening DR was found to increase from $70 \%$ in CKD stage-3 to $82 \%$ in stage- 4 and 5 of CKD and this was statistically significant $(p=0.03)$.

\section{$\underline{\text { Conclusions }}$}

Cystoid morphological pattern followed by mixed type was the most common pattern of DME on OCT found in patients suffering from stage 3 to 5 of CKD. However, the morphological patterns of DME did not significantly vary across various CKD stages. ELM-EZ defects may be considered as an important OCT biomarker for advanced stage of CKD.

\section{Introduction}

Diabetic retinopathy (DR) is one of the leading causes of sight threatening disease in the world. It is estimated that by the year 2030 there would be 366 million diabetics.[1] DR is responsible for $4.8 \%$ of the 37 million cases of blindness throughout the world. The most common cause of vision loss in patients with DR is diabetic macular edema (DME) found in $11.5 \%$, followed by macular ischemia or complications of neovascularization like vitreous hemorrhage or retinal detachment. $[2,3]$ 
An increase in the number of people suffering from diabetes would see a parallel increase in the diabetes associated microvascular complications like retinopathy, nephropathy and neuropathy. These microvascular complications are linked to the duration of diabetes mellitus (DM), poor glycaemic control and systolic hypertension.[3] The presence of DR is a risk factor for development of overt nephropathy whereas overt nephropathy is a significant factor for sight threatening DR.[4] The prevalence of overt diabetic nephropathy is $2.2 \%$.[5] The presence of concomitant nephropathy with retinopathy can lead to early progression of the DME and albuminuria is said to correlate with DME.[6] Penno et al. and Grunwald et al. have shown the association of estimated glomerular filtration rate (eGFR) with DR in type-2 diabetes and demonstrated an independent inverse correlation between eGFR and DR.[7,8] Moderate to severe CKD is estimated to be found in $15-23 \%$ of patients with diabetes.[9] Also the features of diabetic nephropathy occur at a later stage as compared to retinopathy, hence a patient with DR needs to be closely monitored.

Optical Coherence Tomography (OCT) plays an important role in identifying various morphological patterns and prognostic biomarkers in patients with DME.[10,11] The morphological patterns of DME described are diffuse, cystoid, serous retinal detachment (SRD), mixed type and vitreomacular traction (VMT).[11,12] Various prognostic biomarkers such as disorganization of the inner retinal layers (DRIL), hyper reflective dots (HRD) and defects of the external limiting membrane-ellipsoid zone (ELM-EZ) have been described on OCT in eyes with DME. $[13,14]$ OCT provides high resolution images and gives precise information of the morphological pattern and retinal thickness thereby guiding the treatment protocols.

To date, very few studies have been conducted to determine the correlation between the morphological pattern of DME in patients with chronic kidney disease (CKD) secondary to diabetes.[12] The present study was conducted to correlate OCT based morphological patterns of DME and prognostic biomarkers in patients with various stages of CKD secondary to diabetes.

\section{Methods}

It is a multicentric retrospective cross-sectional study conducted at seven centers across India. Review of the medical records was done of patients with a history of DM with deranged renal functions having DR with DME on clinical and OCT evaluation from 1st January 2019 to 31st December 2020. The study followed the declaration of Helsinki and ethical clearance was taken from each centre. A written informed consent was taken from all the patients to use the data for research purpose.

A common excel sheet was shared with all the centres and the following data was captured from the medical records - demographic profile, duration of diabetes, duration of nephropathy, history of hypertension and renal dialysis, renal profile including serum urea, serum creatinine and eGFR. Ophthalmic examination details such as best corrected visual acuity (BCVA), grading of DR (ETDRS grading) and presence of DME were noted.[15] The DR was further grouped into 2 categories for statistical analysis - non sight threatening and sight threatening DR. Non sight threatening DR included 
cases of mild to moderate non-proliferative DR (NPDR). Sight threatening DR included severe NPDR, early and high-risk proliferative DR (PDR).[16]

Patients included were treatment naive DR patients aged $>18$ years with type- 2 diabetes with CKD i.e. serum urea $>40 \mathrm{mg} / \mathrm{dl}$, serum creatinine $>1.6 \mathrm{mg} / \mathrm{dl}$ and eGFR $<60 \mathrm{ml} / \mathrm{min} / 1.73 \mathrm{~m}^{2}$ (systemic condition test done within one month of the OCT performed) having good quality OCT scans and colour fundus photo. One eye of each patient was included having higher foveal sub-field thickness.

eGFR was calculated using CKD-EPI Creatinine Equation (2009) which uses an online eGFR calculator provided by National Kidney Foundation.[17] This calculator requires serum creatinine, age, gender and race of the patient to be entered in the formulae to get the value of eGFR in $\mathrm{ml} / \mathrm{min} / 1.73 \mathrm{~m}^{2}$. Patients were categorized into stages of CKD on the basis of eGFR- Stage 3 Moderate CKD (eGFR $=30-59$ $\mathrm{mL} / \mathrm{min}$ ) Stage 4 Severe CKD (eGFR $=15-29 \mathrm{~mL} / \mathrm{min}$ ) and Stage 5 End Stage CKD (eGFR $<15 \mathrm{~mL} / \mathrm{min})$. [18]

Patients excluded were aged less than 18 years, who had previously undergone laser photocoagulation or intravitreal injection of anti-vascular endothelial growth factor (anti-VEGF) or steroids, as it may alter the morphological pattern of DME, poor quality colour fundus images not allowing the grading of DR and poor image quality of OCT scan. A horizontal raster scan of $12 \times 12 \mathrm{~mm}$ length was taken through the foveal centre. The OCT image at baseline was reviewed for the morphological pattern of DME present and divided into the following 5 types - 1 . spongiform pattern 2. cystoid pattern 3. SRD 4. VMT and 5. mixed type (including both SRD and cystoid pattern) [Figure-1]. The prognostic biomarkers evaluated on OCT were foveal sub-field thickness, HRD, DRIL and ELM-EZ defects. The foveal sub-field thickness was measured manually at the fovea as the distance between the inner limiting membrane and the outer boundary of the retinal pigment epithelium - Bruch's complex using caliper tool of the OCT. [19]

Statistical analyses were performed using $R$ version 4.0.3. The results were expressed as mean $\pm S D$ if the variables were continuous, and as percentage, if categorical. Categorical variables were analyzed using Fisher's exact test and continuous ones using Kruskal Wallis test. P value of $\leq 0.05$ was considered as significant.

\section{Results}

A total of 234 eyes of 234 patients with type-2 diabetes with CKD with DR and DME were included in the study. $175(75 \%)$ males and $59(25 \%)$ female patients were enrolled. Mean duration of DM was 12 years (range 1-40 years). Treatment for DM included insulin in 101 (43\%), oral hypoglycemic agents (OHA) in $110(47 \%)$ and both OHA and insulin therapy in $23(10 \%)$ patients. A total of $19(8 \%)$ out of $234(8 \%)$ of patients were on renal dialysis. $55 \%$ of patients with CKD stage -5 had $\mathrm{HbA} 1 \mathrm{c}$ level $>10 \mathrm{mg} \%$ [Table-1].

\section{Severity of DR and CKD}


Sight threatening DR was found to increase from $70 \%$ in CKD stage-3 to $82 \%$ in stage 4 and 5 of CKD. The results were statistically significant and consistent $(p=0.03)$ [Table-2].

\section{Morphological patterns of DME and CKD}

The most common morphological pattern of DME was cystoid pattern $42 \%$ ( $95 \% \mathrm{Cl}: 36 \%-49 \%$ ) followed by mixed pattern (31\% (95\% Cl: $24 \%-38 \%)$ and then the spongiform pattern in $16 \%$ (95\% Cl: $11 \%-22 \%)$. The SRD and VMT constituted only 6\% (95\% Cl: 0\%-12\%) and 5\% (5\% Cl: 0\%-12\%) respectively. The proportions of different morphological patterns do not significantly vary across various CKD stages ( $p$ $=0.836$, Fisher's Exact Test) [Table-2].

On analyzing the OCT pattern of patients on renal dialysis (19 out of 234 patients) the most common type found was cystoid pattern however this was not statistically significant $(p=0.06)$ [Table-3].

On correlating level of $\mathrm{HbA} 1 \mathrm{c}$ with the morphological pattern of DME it was found that the cystoid pattern on OCT was the most common irrespective of the level of the HbA1c. However, this was not statistically significant $(p=0.15) .50 \%$ of the patients with $\mathrm{HbA} 1 \mathrm{c}>10 \mathrm{mg} \%$ (poorly controlled diabetes) showed VMT [Table-3].

The percentage of sight threatening DR was significantly different across all morphological patterns $(p=0.05)$. The highest percentage risk was seen with VMT $(100 \%)$ followed by SRD $(92 \%)$, spongiform (82\%), mixed (78\%) and minimal in cystoid (69\%) [Table-3].

\section{Visual prognostic biomarkers and stage of CKD}

Detailed distribution of visual prognostic markers with stage of CKD is shown in Table-2. The presence of HRD increased from $68 \%$ in CKD stage-3 and 4 to $83 \%$ patients in CKD stage-5. However, this was found to be statistically not significant $(p=0.13)$. The presence of DRIL was found to have a similar distribution in all the three stages of CKD and it ranged from 43-50\%. Presence of ELM-EZ defects showed a direct correlation with the stage of CKD. They were present in 17 out of 27 patients (63\%) in CKD stage-5 and in 30 out of $52(58 \%)$ and 24 out of 83 patients (29\%) with CKD stage- 4 and stage- 3 respectively. This was found to be statistically significant $(p<0.001)$. A direct correlation was found between foveal sub-field thickness and stage of CKD. Maximum mean foveal sub-field thickness of $524.6 \pm 178.8 \mu \mathrm{m}$ was found in CKD stage- 5 and $463.3 \pm 220 \mu \mathrm{m}$ and $455.82 \pm 166.36 \mu \mathrm{m}$ in CKD stage- 4 and 3 respectively. This was found to be statistically significant ( $p=0.024$, Kruskal Wallis test) [Table-2].

\section{Discussion}

Very few studies have been conducted to determine the correlation of non-ocular factors such as CKD secondary to diabetes with various morphological patterns of DME seen on OCT. [12,20,21] Also the previous studies conducted were single centre studies with a relatively small sample size. None of the studies performed have demonstrated the impact of CKD on the recently described visual prognostic 
biomarkers in DME and correlated them. This study bridges this gap in knowledge which may help in better management.

On review of literature several studies have used proteinuria as a marker of nephropathy and found a relation between disease severity and proteinuria. [12,21,22] However an inconsistent relation has been reported between the two and therefore in our study we have used eGFR as a marker of nephropathy which is possibly a better marker for nephropathy as ethnic variations and age of the patient are used for its determination. Also, eGFR is possibly the earliest marker for nephropathy and albuminuria is an inconsistent marker that may regress or progress. [20,23]

In our group of patients, the average duration of diabetes was 12 years or more with stage 3,4 or 5 CKD and the proportion of different morphological patterns of DME on OCT did not significantly vary across various CKD stages. However, the most common morphological pattern found in the study cohort was cystoid pattern $42 \%$ (95\% Cl: 36-49) followed by mixed pattern 31\% (95\% Cl: $24-38)$ and then the spongiform pattern in $16 \%$ (95\% Cl: 11-22). This is in contrast to what has been reported by Koo et al. in their retrospective study on 93 korean patients of DME with renal dysfunction.[12] They found that the serous macular edema occurred most commonly $(67 \%)$ and this possibly is due to albumin and other extracellular fluid leaking into the sub-retinal space leading to serous detachment.[12] Romero et al. in a series of 112 Spanish patients of type-1 DM of which 23 patients had DR and nephropathy, found spongiform DME to be the most common pattern in 78\% patients(18 out of 23 ) followed by cystoid pattern in 9\%(2 out of 23).[21] Ciardella et al. have reported a case of mixed type (serous and cystoid) macular edema in patients with albuminuria.[24] This shows the variability in the morphological pattern of the DME found on OCT in patients with CKD and no single pattern can be correlated with it.

There is paucity of information in literature regarding the correlation of sight threatening DR with stages of CKD. $[7,8]$ We found sight threatening DR to increase from $70 \%$ in CKD stage-3 to $82 \%$ in CKD stage-4 and 5 . This was found to be statistically significant and consistent $(p=0.03)$. It was also noted that the percentage of sight threatening DR was significantly different across all morphological patterns. The highest percentage risk was seen with VMT (100\%) followed by SRD (92\%), spongiform (82\%), mixed (78\%) and minimal in cystoid (69\%). This highlights the fact that with advancement in the stage of CKD the patient has an increased risk of loss of vision.

In our study on subgroup analysis of patients on renal dialysis (19 out of 234 patients) spongiform pattern was not found in any patient and cystoid pattern was the commonest though not found to be statistically significant. This is in contrast to what has been reported by Takamura et al. who in their series of 118 eyes of patients on hemodialysis found spongiform pattern of DME to be the most common pattern in $53 \%$ (62 out of 118 eyes).[25]

Longer duration of DM, age, hypercholesterolemia, poor glycaemic control (elevated $\mathrm{HbA1C}$ ), hypertension and the use of insulin are regarded as major risk factors for manifestation of DME.[3] In our study glycosylated haemoglobin ( $\mathrm{HbA1c}$ ) was found to be in the higher range $>10 \mathrm{mg} \%$ in patients with 
more advanced stage of CKD and on correlating level of HbA1c with the morphological pattern of DME. it was found that cystoid pattern was the most common irrespective of the level of the HbA1c. However, this was not found to be statistically significant. An observation was made that $50 \%$ of the patients with $\mathrm{HbA} 1 \mathrm{c}>10 \mathrm{mg} \%$ (poorly controlled diabetes) showed VMT. This possibly could be due to the primary insult of hyperglycemia leading to the development and acceleration of advanced glycation end products (AGEs) which promotes leukostasis and cause damage to both pericytes and endothelial cells. They are also believed to promote mechanical changes in the vitreous and at the vitreous-retinal interface promoting VMT and subsequent DME. $[26,27]$ This highlights the fact that the patients with poorly controlled diabetes are likely to have more VMT causing a higher risk of sight threatening DR.

To the best of our knowledge there is a lacunae in literature regarding the impact of CKD on various OCT biomarkers. In our study on correlating the OCT biomarkers with stage of CKD we found ELM-EZ defects showed a direct correlation with the stage of CKD. They were present in 17 out of 27 patients $(63 \%)$ in CKD stage -5 and in 30 out of 52 (58 \%) and 24 out of 83 patients (29\%) with CKD stage- 4 and stage- 3 respectively. This was found to be highly statistically significant. DRIL and HRD showed no correlation. This suggests that patients suffering from diabetes and end stage CKD show more ELM-EZ defects on OCT causing poorer visual prognosis. In a patient suffering from diabetes if on evaluation of OCT one finds ELM-EZ defects then it is important to check the renal function in such patients, if they are not aware of it.

Knudsen et al. studied DME in 20 patients with type-2 DM and found a strong correlation between increased retinal thickness on OCT and increased urinary albumin excretion rate.[6] Acan D et al. in their study of 53 patients with DME found that diffuse retinal thickness (62\%) was much higher with micro or macro albuminuria compared to cystoid macular edema (42\%) and SRD $(21 \%)$. They also found that the $\mathrm{HbA1c}$ levels were higher in the diffuse retinal thickness group.[28] We found a direct correlation between foveal subfield thickness and stage of CKD. Maximum mean foveal sub-field thickness of $524.6 \pm 178.8$ $\mu \mathrm{m}$ was found in CKD stage- 5 . It was noted to be $463.3 \pm 220 \mu \mathrm{m}$ and $455.82 \pm 166.36 \mu \mathrm{m}$ in CKD stage4 and 3 respectively. This was found to be statistically significant.

All the previous studies conducted were single centre studies with a relatively small sample size. The present study is the first from the Indian subcontinent and the largest multicentric series till date. It is also the first study to correlate eGFR with the morphological pattern of DME, sight threatening DR and OCT biomarkers with stage of CKD. The limitations of this study are as follows- it is a retrospective study, there was no control group with no CKD for comparison, the sample size of patients on renal dialysis was small to draw conclusions thereby requiring a further study with larger sample size.

\section{Conclusion}

Cystoid morphological pattern followed by mixed type was the most common pattern of DME on OCT found in patients with stage 3 to 5 of CKD. However, the morphological patterns of DME did not significantly vary across various CKD stages. The risk of sight threatening DR increases with the increase 
in the stage of CKD requiring an early referral to an ophthalmologist for a close monitoring of DR. ELM-EZ defects maybe considered as an OCT biomarker for advanced stage of CKD and we urge clinicians to pay close attention to this finding on OCT in order to timely evaluate the renal parameters and refer to a nephrologist.

\section{Declarations}

Funding/Financial support: Clinical Research Department, Dr Shroff's Charity Eye Hospital (MA,MS,SS),India and Ornate India GCRF UKRI funding (Grant No: MR/P207881/1), UK.

We have no financial interest to disclose.

Conflicts of interest/Competing interests: We have no conflict of interest with each other.

\section{Availability of data and material: The data is available for referencing if needed.}

This manuscript has not been published elsewhere and it has not been submitted simultaneously for publication elsewhere.

The manuscript has been read and approved by all the authors.

\section{References}

1. Aiello LP, Gardner TW, King GL, et al. Diabetic retinopathy. Diabetes Care. 1998 Jan;21(1):14356. https://doi.org/10.2337/diacare.21.1.143.

2. Raman R, Ganesan S, Pal SS, et al. Incidence and Progression of Diabetic Retinopathy in Urban India: Sankara Nethralaya-Diabetic Retinopathy Epidemiology and Molecular Genetics Study (SN-DREAMS II), Report 1. Ophthalmic Epidemiol. 2017 Oct;24(5):294-

302. https://doi.org/10.1080/09286586.2017.1290257

3. Klein R, Klein BE, Moss SE, et al. The Wisconsin epidemiologic study of diabetic retinopathy. IV. Diabetic macular edema. Ophthalmology. 1984 Dec;91(12):1464-74. https://doi.org/10.1016/s01616420(84)34102-1.

4. Romero-Aroca P, Baget-Bernaldiz M, Reyes-Torres J, et al. Relationship between diabetic retinopathy, microalbuminuria and overt nephropathy, and twenty-year incidence follow-up of a sample of type 1 diabetic patients. J Diabetes Complications. 2012 Nov-Dec;26(6):506-

12. https://doi.org/10.1016/j.jdiacomp.2012.06.010.

5. Unnikrishnan RI, Rema M, Pradeepa R, et al. Prevalence and risk factors of diabetic nephropathy in an urban South Indian population: the Chennai Urban Rural Epidemiology Study (CURES 45).

Diabetes Care. 2007 Aug;30(8):2019-24. https://doi.org/10.2337/dc06-2554.

6. Knudsen ST, Bek T, Poulsen PL, et al. Macular edema reflects generalized vascular hyperpermeability in type 2 diabetic patients with retinopathy. Diabetes Care. 2002 Dec;25(12):2328-34. 
https://doi.org/ 10.2337/diacare.25.12.2328.

7. Penno G, Solini A, Zoppini G, et al. Renal Insufficiency And Cardiovascular Events (RIACE) Study Group. Rate and determinants of association between advanced retinopathy and chronic kidney disease in patients with type 2 diabetes: the Renal Insufficiency And Cardiovascular Events (RIACE) Italian multicenter study. Diabetes Care. 2012 Nov;35(11):2317-23. https://doi.org/ 10.2337/dc120628.

8. Grunwald JE, Alexander J, Ying GS, et al. CRIC Study Group. Retinopathy and chronic kidney disease in the Chronic Renal Insufficiency Cohort (CRIC) study. Arch Ophthalmol. 2012 Sep;130(9):113644. https://doi.org/10.1001/archophthalmol.2012.1800.

9. Middleton RJ, Foley RN, Hegarty J, et al. The unrecognized prevalence of chronic kidney disease in diabetes. Nephrol Dial Transplant. 2006 Jan;21(1):88-92. https://doi.org/ 10.1093/ndt/gfi163.

10. Kang SW, Park CY, Ham DI. The correlation between fluorescein angiographic and optical coherence tomographic features in clinically significant diabetic macular edema. Am J Ophthalmol. 2004 Feb;137(2):313-22. https://doi.org/10.1016/j.ajo.2003.09.016.

11. Otani T, Kishi S, Maruyama Y. Patterns of diabetic macular edema with optical coherence tomography. Am J Ophthalmol. 1999 Jun;127(6):688-93. https://doi.org/10.1016/s00029394(99)00033-1.

12. Koo NK, Jin HC, Kim KS, et al. Relationship between the morphology of diabetic macular edema and renal dysfunction in diabetes. Korean J Ophthalmol. 2013 Apr;27(2):98-102. https://doi.org/10.3341/kjo.2013.27.2.98.

13. Sun JK, Lin MM, Lammer J, et al. Disorganization of the retinal inner layers as a predictor of visual acuity in eyes with center-involved diabetic macular edema. JAMA Ophthalmol. 2014 Nov;132(11):1309-16. https://doi.org/10.1001/jamaophthalmol.2014.2350.

14. Das R, Spence G, Hogg RE, et al. Disorganization of Inner Retina and Outer Retinal Morphology in Diabetic Macular Edema. JAMA Ophthalmol. 2018 Feb 1;136(2):202-208. https://doi.org/10.1001/jamaophthalmol.2017.6256.

15. Grading diabetic retinopathy from stereoscopic color fundus photographs--an extension of the modified Airlie House classification. ETDRS report number 10. Early Treatment Diabetic Retinopathy Study Research Group. Ophthalmology. 1991 May;98(5 Suppl):786-806.

16. Klein R, Klein BE, Magli YL, et al. An alternative method of grading diabetic retinopathy. Ophthalmology. 1986 Sep;93(9):1183-7. https://doi.org/10.1016/s0161-6420(86)33606-6.

17. National Kidney Foundation.eGFR calculator; c2016 [cited 2016 Nov 13]. Available from: https://www.kidney.org/apps/professionals/egfr-calculator

18. Chapter 1: Definition and classification of CKD. Kidney Int Suppl (2011). 2013 Jan;3(1):19-62.

19. Falcão MS, Gonçalves NM, Freitas-Costa P, et al. Choroidal and macular thickness changes induced by cataract surgery. Clin Ophthalmol. 2014;8:55-60. https://doi.org/10.2147/OPTH.S53989.

20. Temkar S, Karuppaiah N, Takkar B, et al. Impact of estimated glomerular filtration rate on diabetic macular edema. Int Ophthalmol. 2018 Jun;38(3):1043-1050. https://doi.org/10.1007/s10792-017- 
0557-8.

21. Romero P, Baget M, Mendez I, et al. Diabetic macular edema and its relationship to renal microangiopathy: a sample of Type I diabetes mellitus patients in a 15-year follow-up study. J Diabetes Complications. 2007 May-Jun;21(3):172-80. https://doi.org/10.1016/j.jdiacomp.2006.07.008.

22. Zhuang $X$, Cao D, Zeng $Y$, et al. Associations between retinal microvasculature/microstructure and renal function in type 2 diabetes patients with early chronic kidney disease. Diabetes Res Clin Pract. 2020 Oct;168:108373. https://doi.org/10.1016/j.diabres.2020.108373.

23. Man RE, Sasongko MB, Wang JJ, et al. The Association of Estimated Glomerular Filtration Rate With Diabetic Retinopathy and Macular Edema. Invest Ophthalmol Vis Sci. 2015 Jul;56(8):4810-6. https://doi.org/10.1167/iovs.15-16987.

24. Ciardella AP. Partial resolution of diabetic macular oedema after systemic treatment with furosemide. Br J Ophthalmol. 2004 Sep;88(9):1224-5. https://doi.org/10.1136/bjo.2004.042580.

25. Takamura Y, Matsumura T, Ohkoshi K, et al. Functional and anatomical changes in diabetic macular edema after hemodialysis initiation: One-year follow-up multicenter study. Sci Rep. 2020 May 8;10(1):7788. https://doi.org/10.1038/s41598-020-64798-4.

26. Bhagat N, Grigorian RA, Tutela A, et al. Diabetic macular edema: pathogenesis and treatment. Surv Ophthalmol. 2009 Jan-Feb;54(1):1-32. https://doi.org/10.1016/j.survophthal.2008.10.001.

27. Joussen AM, Murata T, Tsujikawa A, et al. Leukocyte-mediated endothelial cell injury and death in the diabetic retina. Am J Pathol. 2001 Jan;158(1):147-52. https://doi.org/10.1016/S00029440(10)63952-1.

28. Acan D, Karahan E, Kocak N, et al. Evaluation of systemic risk factors in different optical coherence tomographic patterns of diabetic macular edema. Int J Ophthalmol. $2018 \mathrm{Jul}$ 18;11(7):1204-1209. https://doi.org/10.18240/ijo.2018.07.21. eCollection 2018.

\section{Tables}

Table-1: Demographic data of study cohort 


\begin{tabular}{|c|c|c|c|c|c|}
\hline \multicolumn{4}{|c|}{ CKD } & \multirow{3}{*}{$\begin{array}{l}\text { Total } \\
\mathrm{n}(\%)\end{array}$} & \multirow[t]{3}{*}{ p value } \\
\hline \multirow[t]{2}{*}{ Sex } & Stage-3 & Stage-4 & Stage-5 & & \\
\hline & $n(\%)$ & $n(\%)$ & $n(\%)$ & & \\
\hline Male & $96(82)$ & $47(63)$ & $32(76)$ & $175(75)$ & \multirow[t]{3}{*}{0.011} \\
\hline Female & $21(18)$ & $28(37)$ & $10(24)$ & $59(25)$ & \\
\hline Total & 117 & 75 & 42 & 234 & \\
\hline \multicolumn{6}{|l|}{ Systemic treatment } \\
\hline Insulin & $51(42)$ & $34(44)$ & $16(43)$ & $101(43)$ & \multirow[t]{4}{*}{0.982} \\
\hline $\mathrm{OHA}$ & $56(47)$ & $35(45)$ & $19(52)$ & $110(47)$ & \\
\hline Mixed (Insulin and OHA) & $13(11)$ & $8(11)$ & $2(5)$ & $23(10)$ & \\
\hline Total & 120 & 77 & 37 & 234 & \\
\hline \multicolumn{6}{|l|}{$\mathrm{HbA1c}(\mathrm{mg} \%)$} \\
\hline$\leq 8$ & $44(38)$ & $33(44)$ & $15(36)$ & $92(39)$ & \multirow[t]{4}{*}{0.127} \\
\hline$>8-\leq 10$ & $25(21)$ & $18(24)$ & $4(9)$ & $47(20)$ & \\
\hline$>10$ & $48(41)$ & $24(32)$ & $23(55)$ & $95(41)$ & \\
\hline Total & 117 & 75 & 42 & 234 & \\
\hline \multicolumn{6}{|l|}{ Renal Dialysis } \\
\hline Yes & $1(1)$ & $1(1)$ & $17(40)$ & $19(8)$ & \multirow[t]{3}{*}{$<0.001$} \\
\hline No & 116(99) & $74(99)$ & $25(60)$ & $215(92)$ & \\
\hline Total & 117 & 75 & 42 & 234 & \\
\hline
\end{tabular}

CKD- chronic kidney disease, HbA1c- glycated hemoglobin, mg- milligram, n- number, OHA- oral hypoglycemic agents

Table-2: Correlation of various OCT parameters with CKD staging 
Morphological pattern

CKD

Total

P

Stage-3 Stage-4 Stage-5 $n(\%)$

value

$\mathrm{n}(\%)$

$n(\%)$

$n(\%)$

Spongiform Pattern

21(18)

12(16)

$5(12)$

$38(16)$

0.836

Cystoid Pattern

$50(43)$

27(36)

22(53)

99(42)

SRD

6(5)

6(8)

1(2)

13(6)

VMT

6(5)

3(4)

3(7)

12(5)

Mixed Type (cystoid and SRD)

34(29)

27(36)

11(26)

72(31)

Total

117

75

42

234

\section{Grading of DR}

\begin{tabular}{|c|c|c|c|c|c|c|}
\hline \multicolumn{2}{|c|}{ Non sight threatening } & $35(30)$ & $10(13)$ & $10(24)$ & $55(23)$ & \multirow[t]{3}{*}{0.03} \\
\hline \multicolumn{2}{|l|}{ Sight threatening } & $82(70)$ & $65(87)$ & $32(76)$ & $179(77)$ & \\
\hline \multicolumn{2}{|l|}{ Total } & 117 & 75 & 42 & 234 & \\
\hline \multicolumn{7}{|l|}{ OCT biomarkers } \\
\hline \multirow[t]{2}{*}{ HRD } & Present & $79(68)$ & $51(68)$ & $35(83)$ & $165(71)$ & \multirow[t]{2}{*}{0.13} \\
\hline & Absent & $38(32)$ & $24(32)$ & $7(17)$ & $69(29)$ & \\
\hline \multirow[t]{2}{*}{ DRIL } & Present & $52(44)$ & $32(43)$ & $21(50)$ & $105(45)$ & \multirow[t]{2}{*}{0.73} \\
\hline & Absent & $65(56)$ & $43(57)$ & $21(50)$ & $129(55)$ & \\
\hline \multirow[t]{2}{*}{ ELM-EZ defect } & Present & $24(29)$ & $30(58)$ & $17(63)$ & 71(44) & \multirow[t]{2}{*}{$<0.001$} \\
\hline & Absent & $59(71)$ & $22(42)$ & $10(37)$ & $91(56)$ & \\
\hline \multicolumn{2}{|c|}{$\begin{array}{l}\text { Foveal Subfield Thickness }(\mu \mathrm{m}) \\
\text { (Mean } \pm \text { SD) }\end{array}$} & $\begin{array}{l}455.82 \pm \\
166.36\end{array}$ & $\begin{array}{l}463.36 \pm \\
220.7\end{array}$ & $\begin{array}{l}524.6 \pm \\
178.8\end{array}$ & $\begin{array}{l}470.58 \pm \\
188.48\end{array}$ & 0.024 \\
\hline
\end{tabular}

CKD- chronic kidney disease, DR- diabetic retinopathy, DRIL- disorganization of the inner retinal layers, ELM-EZ- external limiting membrane-ellipsoid zone, HRD- hyper reflective dots, $\mu$ m- micrometre, $\mathrm{n}$ - number, OCT- optical coherence tomography, SD- standard deviation, SRD- serous retinal detachment, VMT- vitreomacular traction

Table-3: Correlation of severity of DR, glycemic control and renal dialysis with morphological pattern of DME on OCT 
Morphological pattern of DME on OCT

\begin{tabular}{|c|c|c|c|c|c|c|c|}
\hline Grading of DR & $\begin{array}{l}\text { Spongiform } \\
\text { Pattern } \\
\mathrm{n}(\%)\end{array}$ & $\begin{array}{l}\text { Cystoid } \\
\text { Pattern } \\
\mathrm{n}(\%)\end{array}$ & $\begin{array}{l}\text { SRD } \\
n(\%)\end{array}$ & $\begin{array}{l}\text { VMT } \\
\text { n(\%) }\end{array}$ & $\begin{array}{l}\text { Mixed } \\
\text { Type } \\
\mathrm{n}(\%)\end{array}$ & $\begin{array}{l}\text { Total } \\
\mathrm{n}(\%)\end{array}$ & $\begin{array}{l}p \\
\text { value }\end{array}$ \\
\hline $\begin{array}{l}\text { Non sight } \\
\text { threatening }\end{array}$ & $7(18)$ & $31(31)$ & $1(8)$ & $0(0)$ & $16(22)$ & $55(23)$ & 0.05 \\
\hline $\begin{array}{l}\text { Sight } \\
\text { threatening }\end{array}$ & $31(82)$ & 68(69) & 12(92) & $12(100)$ & $56(78)$ & 179(77) & \\
\hline Total & 38 & 99 & 13 & 12 & 72 & 234 & \\
\hline \multicolumn{8}{|l|}{ HbA1c (mg\%) } \\
\hline$\leq 8$ & $16(42)$ & $43(44)$ & $4(31)$ & $4(33)$ & $25(35)$ & $92(39)$ & \multirow[t]{4}{*}{0.15} \\
\hline$>8-\leq 10$ & $9(24)$ & 15(15) & $7(54)$ & $2(17)$ & 14(19) & $47(21)$ & \\
\hline$>10$ & 13(34) & $41(41)$ & $2(15)$ & $6(50)$ & $33(46)$ & $95(40)$ & \\
\hline Total & 38 & 99 & 13 & 12 & 72 & 234 & \\
\hline \multicolumn{8}{|l|}{ Renal dialysis } \\
\hline Yes & $0(0)$ & 12(12) & $1(8)$ & $2(17)$ & $4(6)$ & $19(8)$ & \multirow[t]{3}{*}{0.06} \\
\hline No & $38(100)$ & 87(88) & 12(92) & 10(83) & $68(94)$ & 215(92) & \\
\hline Total & 38 & 99 & 13 & 12 & 72 & 234 & \\
\hline
\end{tabular}

DME- diabetic macular edema, DR- diabetic retinopathy, HbA1c- glycated hemoglobin, mg- milligram, n- number, OCT- optical coherence tomography, SRD- serous retinal detachment, VMT- vitreomacular traction

Figures 


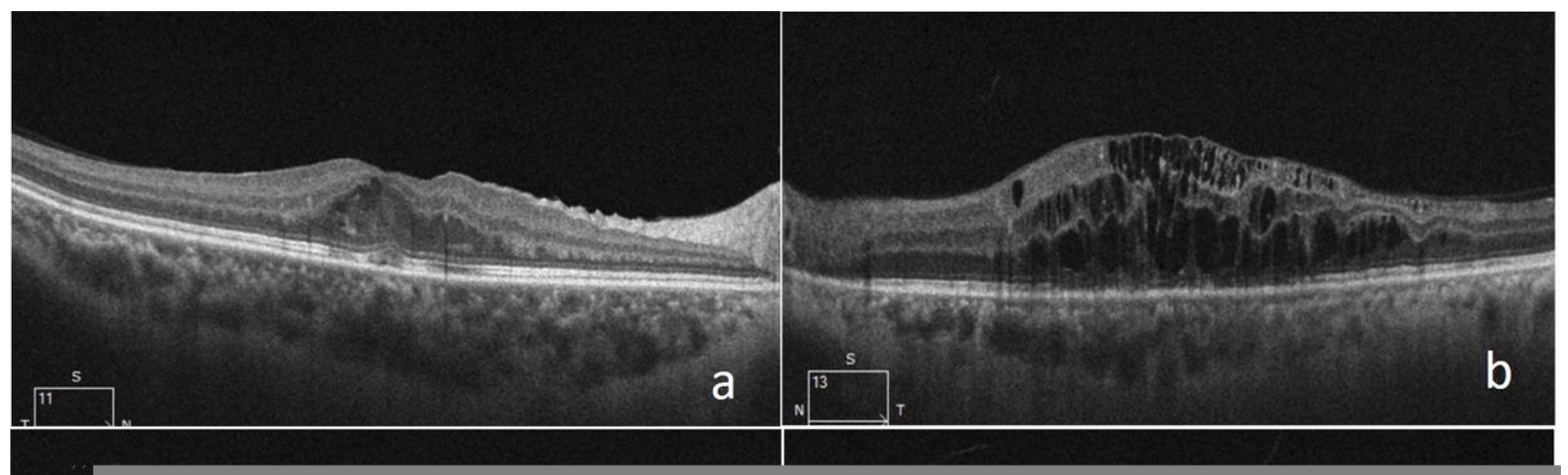

\section{Figure 1}

Five different morphological patterns of diabetic macular edema on optical coherence tomography. (a) Spongiform type (b) Cystoid type (c) Serous retinal detachment type (d) Vitreomacular tractional type and (e) Mixed type (serous + cystoid) 